\title{
Know your market
}

\author{
The photonic sensors market is a diverse and fragmented one. David Krohn, chair of the Optoelectronics Industry \\ Development Association's Photonic Sensor Consortium, tells Nadya Anscombe that the difference between \\ success and failure often depends on understanding your market.
}

\begin{abstract}
How would you describe the photonic sensors market?

There are basically two types of photonic sensors: point sensors and distributed sensors. Point sensors have a variety of different forms, including single-point fibre sensors, optical chip sensors and spectrometers. Distributed sensors are usually fibre-based, but some companies are developing distributed sensor networks based on microelectromechanical and wireless systems.
\end{abstract}

What are the trends in the distributed fibre sensor market?

The annual market for distributed fibre sensors is estimated to be worth US $\$ 550$ million, and is much bigger than the market for point sensors. This is because distributed sensing technology is used to measure temperature and strain in the oil and gas industry, in which operating conditions are harsh and other sensor technologies simply cannot cope.

The recent oil spill in the Gulf of Mexico has highlighted the fact that oil wells need to get a lot smarter, and there are opportunities here for fibre sensor technologies. As well as safety, sensor technology can also improve the efficiency of oil wells. A 'dumb' well is only about $30 \%$ efficient, with $70 \%$ of the available oil left underground because it is too difficult to locate or extract. Fibre sensors can be used to locate oil reservoirs and monitor their conditions. They also enable companies to go back to old wells and extract more oil.

There are essentially four types of distributed fibre sensor technology, based on either fibre Bragg gratings, Brillouin scattering, Raman scattering or interferometric approaches.

Distributed networks based on fibre Bragg gratings are essentially a network of point sensors, and have found applications in many areas including the monitoring of strain for smart structures and smart oil wells. Brillouin scattering technology is newer and requires a more expensive interface system than fibre Bragg grating technology. However, the whole fibre acts as the sensor and it can be used to measure strain as well as temperature. Raman scattering technology is currently the dominant photonic sensor technology for measuring temperature in

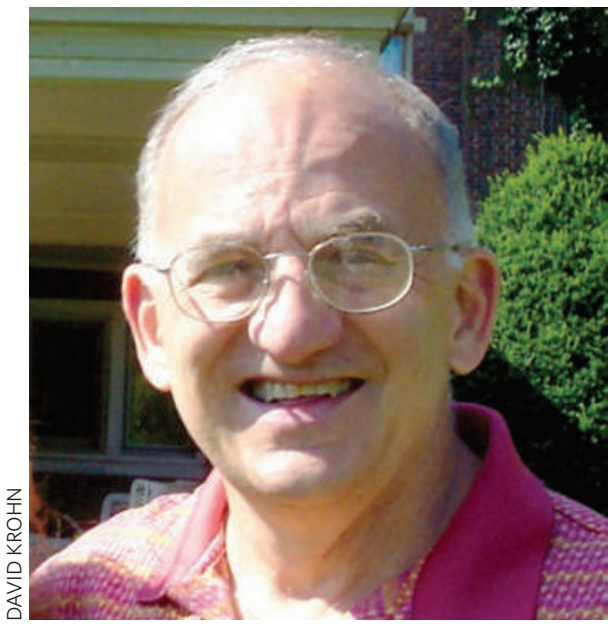

David Krohn: "My estimate is that $70 \%$ of company failures in the sensors market are not down to the technology, but are because the manufacturer did not understand their market properly."

the oil and gas industry because it allows continuous runs of $15 \mathrm{~km}$ or more - typical in many oil well applications. Several interferometric distributed fibre sensor technologies exist, and all are very sensitive to vibrations. These are used in military applications and in seismic sensing.

What are the current trends in the point sensor market?

The biggest application for point sensors is in optical gyroscopes, which, although expensive, have many advantages over other technologies. For example, they have no moving parts and thus tend not to fail. Other emerging and exciting uses for point sensors include applications in the medical market such as internal body probes for temperature and pressure detection. The challenge in this industry is to make sensors cheaply enough so that they can be disposable.

Where are the big market opportunities? The biggest opportunities for point sensors will be in patient diagnostics and patient monitoring, as well as in drug discovery. I also believe that the energy market holds many opportunities for photonic sensors. For example, real-time continuous spectroscopy can minimize slagging in the coal industry; fibre sensors can be used for power grid monitoring or hot-spot detection in power lines; and Raman scattering can be used to monitor geothermal wells, which are similar in construction to those in the oil and gas industry but require higher temperature sensors. This is an emerging application, but strong growth is expected by 2011.

I also believe that light detection and ranging (LIDAR) technology can be combined with integrated strain sensors in wind turbines to improve the efficiency of these enormous structures. The resulting system can be used to detect and anticipate wind direction, providing information that can then be used to move the turbine blades into the correct position to minimize blade stress and increase power output.

What are the main challenges that the photonic sensor industry faces today?

When companies are developing new sensor technologies, it is essential that they have a good grasp of the markets in which their technology can be used. I sit on a review board that makes decisions on funding projects, and I have seen many companies that simply do not understand their markets. In the sensor industry, every market is different - you cannot sell the same sensor to the food industry and to the oil and gas industry, for example. It is difficult for companies to diversify, and many firms become pure players with products aimed at only one market. This can be a dangerous strategy. My estimate is that $70 \%$ of company failures in the sensors market are not down to the technology, but are because the manufacturer did not understand their market properly. The other challenges are cost and the creation of standards and legislation. As technologies mature and systems become standardized, costs come down. There is already a lot of activity in creating standards and legislation. The picture has certainly improved compared with a couple of years ago.

INTERVIEW BY NADYA ANSCOMBE

Nadya Anscombe is a freelance journalist based in the United Kingdom. 